\title{
TIME SCALES AND MASSES OF OH-EMISSION REGIONS
}

\author{
T. K. MENON
}

Tata Institute of Fundamental Research, Bombay, India

\begin{abstract}
The velocity structure of $\mathrm{OH}$ profiles of $\mathrm{OH}$ maser regions is used to estimate the time scales of the source distributions. Time scales are found to be of the order of a few thousand years in typical cases. The velocity dispersions and intensity distribution of components are interpreted in terms of a collapsing cloud model of the emission regions. The masses of the clouds are found to be in the range from a few solar masses to a few hundred solar masses for the well-known emission regions.
\end{abstract}

\section{(The following summary was prepared from the tapes by the Editors.)}

A number of $\mathrm{OH}$ maser sources were investigated, in which the scale size is known from VLBI or high-resolution observations. The velocity structure of the $\mathrm{OH}$ profiles was used to estimate the time scales of the source distributions. For all groups of $\mathrm{OH}$ sources that are as wide in angular size as the Orion or W3 groups, the time scales are found to be on the order of a few thousand years in typical cases.

While investigating this time scale a peculiarity was noticed in the intensity distribution of the spectral components. Orion shows a typical spectrum of the multicomponent $\mathrm{OH}$ sources. W3 and W49 are other examples. The Orion $\mathrm{OH}$ spectrum consists of 20 or more well-separated emission components, covering the velocity range -11 to $+23 \mathrm{~km} \mathrm{~s}^{-1}$. (The velocity of the local standard of rest at Orion is $\sim+8 \mathrm{~km} \mathrm{~s}^{-1}$.) Starting at the more negative velocities, the intensity of the components increases, building up to very large intensities toward positive velocities. The component at the highest velocity on the positive side has the largest intensity. At still higher velocities nothing more is seen. All the low intensity components are to the negative velocity side of the high intensity components.

Since this happens in a large number of multi-component sources it may imply that there is a cutoff velocity at the positive velocity side. This may be a velocity of escape, or it may be a terminal velocity of infalling material. In either case one can estimate the mass. I have made a collapsing cloud model of the emission regions. The masses of the clouds are found to be in the range from a few solar masses to a few hundred solar masses for the well known emission sources.

T. K. Menon

Tata Institute of Fundamental Research, Homi Bhabha Road, Bombay 400 005, India

\section{DISCUSSION}

Mezger: Do you apply Zeeman analysis to the W49 region? Can you estimate a mass?

Menon: Not Zeeman analysis, but just the fact that the line profile has its highest intensity at the 
positive velocities, with a falling off of intensity toward lower velocities. I have not computed a mass for W49.

Morimoto: Davies stated the W3 OH cluster can be supported by a star of $20 M_{\odot}$. Your mass of $600 M_{\odot}$ seems to contradict this.

Menon: My estimate of $600 M_{\odot}$ refers to the Orion $\mathrm{OH}$ cluster. The mass for $\mathrm{W} 3 \mathrm{OH}$ turns out to be about $15 M_{\odot}$ since the velocity spread is only about $2 \mathrm{~km} \mathrm{~s}^{-1}$.

Davies: You based your estimates of cloud masses on infall velocities of approximately $20 \mathrm{~km} \mathrm{~s}^{-1}$. In the case of $\mathrm{W} 3 \mathrm{OH}$, where the structure of the $\mathrm{OH}$ object has been investigated in detail by interferometry, the spread in velocity is $\pm 2 \mathrm{~km} \mathrm{~s}^{-1}$ when account is taken of the Zeeman splitting. This velocity leads to a much lower estimate of the object.

Menon: My estimate of mass refers to the Orion $\mathrm{OH}$ cluster. In the case of $\mathrm{W} 3 \mathrm{OH}$ since the velocity spread is small the corresponding mass will also be small since the mass is proportional to the square of the velocity.

Townes: In some of these profiles I think I can see several weak components at higher velocities than that of the strongest peak.

Menon: There may be a few very weak components beyond, but there is a piling up of material at this terminal velocity. 